\title{
Efficacy and safety of vinorelbine and cisplatin regimen of different doses and intensities for neoadjuvant chemotherapy in patients with locally advanced esophageal carcinoma
}

\author{
Ke Jin ${ }^{1,2 \#}$, Baofu Chen ${ }^{1,2 \#}$, Chunguo Wang ${ }^{1,2}$, Bo Zhang ${ }^{1,2}$, Jian Zhang ${ }^{1,2}$, Min Kong ${ }^{1,2}$, Linyao Wang ${ }^{1,2}$, \\ Chengchu Zhu ${ }^{1,2 *}$, Jianfei Shen ${ }^{1,2 *}$ \\ ${ }^{1}$ Department of Cardiothoracic Surgery, Taizhou Hospital of Zhejiang Province Affiliated to Wenzhou Medical University, Taizhou, China; ${ }^{2}$ Key \\ Laboratory of Minimally Invasive Techniques \& Rapid Rehabilitation of Digestive System Tumor of Zhejiang Province, Taizhou, China \\ Contributions: (I) Conception and design: J Shen, C Zhu; (II) Administrative support: B Chen, C Zhu; (III) Provision of study materials or patients: \\ All authors; (IV) Collection and assembly of data: K Jin, L Wang; (V) Data analysis and interpretation: K Jin, B Chen, C Wang, B Zhang, J Zhang, M \\ Kong, L Wang; (VI) Manuscript writing: All authors; (VII) Final approval of manuscript: All authors. \\ \#These authors contributed equally to this work. \\ *These authors contributed equally to this work as senior authors. \\ Correspondence to: Mr. Chengchu Zhu, MD; Jianfei Shen, PhD. Taizhou Hospital of Zhejiang Province Affiliated to Wenzhou Medical University, \\ 150\# Ximen Street Linhai District, Taizhou 317000, China. Email: zhucc@enzemed.com; jianfei051@163.com.
}

Background: There are few studies focused on comparing the toxicity, postoperative complication rate, and survival among patients with locally advanced esophageal squamous cell cancer receiving a different dose and intensity of vinorelbine plus cisplatin for neoadjuvant chemoradiotherapy (nCRT) followed by surgery.

Methods: In total, 78 patients diagnosed with locally advanced esophageal squamous cell cancer that had received a vinorelbine and cisplatin (VP)1 or VP2 regimen for nCRT followed by surgery in Taizhou Hospital of Zhejiang Province between June 2008 and December 2016 were retrospectively analyzed. The VP1 regimen involved cisplatin $75 \mathrm{mg} / \mathrm{m}^{2}$ on day 1 , and vinorelbine $25 \mathrm{mg} / \mathrm{m}^{2}$ on days 1 and 8 , for two cycles. The VP2 regimen involved cisplatin $25 \mathrm{mg} / \mathrm{m}^{2}$ on days 1 to 4 , and vinorelbine $25 \mathrm{mg} / \mathrm{m}^{2}$ on days 1 and 8 , for two cycles. The rate of adverse events, postoperative complications, and survival were compared between the two groups.

Results: The median overall survival (OS) was 97.6 months (85.6-109.7) in the VP2 group, which was not significantly different to that of the VP1 group [hazard ratio (HR), 1.008 (0.999-1.108); P=0.509]. The main toxicity was hematologic adverse events. The VP2 group had significantly higher rates of all grades of anemia, leukopenia, neutropenia, and thrombocytopenia (all $\mathrm{P}<0.05$ ), as well as grade 3 or 4 of leukopenia and neutropenia $(\mathrm{P}<0.05)$ compared to the VP1 group. Regarding postoperative complications, the VP2 group had a significantly higher rate of pulmonary infection than the VP1 group $(\mathrm{P}<0.05)$.

Conclusions: Compared with VP2, VP1 showed comparable efficacy in terms of survival, with less hematologic toxicity and postoperative pulmonary infection. Therefore, we recommended that VP1 over VP2 to be the optimized VP neoadjuvant chemotherapy regimen for locally advanced esophageal squamous cell cancer.

Keywords: Esophageal squamous cell cancer; neoadjuvant chemoradiotherapy (nCRT); surgery; chemotherapy regimen; overall survival (OS)

Submitted Dec 30, 2020. Accepted for publication Apr 22, 2021.

doi: $10.21037 / \mathrm{atm}-21-458$

View this article at: http://dx.doi.org/10.21037/atm-21-458 


\section{Introduction}

China has the highest incidence rate of esophageal cancer (EC) and largest number of EC patients anywhere in the world (1). Most newly diagnosed esophagus cancer patients present with locally advanced disease. For this patient population, surgery is the mainstay treatment choice (2). The addition of neoadjuvant chemoradiotherapy (nCRT) has also been a standard treatment for locally advanced $\mathrm{EC}$ in many regions to improve the outcome of EC treatment (3). Theoretically, nCRT could improve local symptoms, reduce micro-metastasis, and prolong survival (3-5), and is thus a promising EC treatment pattern. Significant efforts have been exerted for further exploration of this therapy modality. However, nCRT-related toxicity and increased postoperative complications and mortality might be major problems that would limit the benefit of nCRT (6). Patients with esophageal squamous cell carcinoma (ESCC), and particularly those with advanced disease, tend to be poorly tolerant to chemotherapy due to the heavy use of tobacco and alcohol (6). In some studies, the chemotherapy-toxicity related deaths rates reached $10-14 \%$ among ESCC patients $(7,8)$. According to the meta-analysis by Fiorica et al., nCRT plus surgery increased postoperative mortality [odds ratio (OR) 2.10; $\mathrm{P}=0.01$ ] compared with surgery alone (9). In one randomized study among EC patients, cisplatin-5-fluorouracil (FU) for eight cycles conferred no benefits on survival, but had more complications compared to no chemotherapy (10). Therefore, optimizing the dose and intensity, especially with regards to toxicity, is crucial in nCRT for ESCC.

The cisplatin-based chemotherapy regimen is the most often used regimen in EC treatment (11). Different combinations of platinum compounds and other chemotherapy agents had been examined in EC treatment (12). Vinorelbine is a semisynthetic vinca alkaloid that exhibits activity and low toxicity in ESCC treatment (13-15). When vinorelbine was combined with cisplatin, the two agents acted synergistically and showed superior tolerance and efficacy. In a study by Conroy et al., the combination of vinorelbine and cisplatin (VP) was used to treat 71 metastatic ESCC patients. The response rate of the regimen was $33.8 \%$, and the median survival was 6.8 months (6). VP neoadjuvant chemotherapy was extensively acknowledged to be sensitive for EC (16-19). Liu et al. compared VP and cisplatin/FU for neoadjuvant chemotherapy in 114 patients with locally advanced ESCC in a retrospective matched casecontrol study. In their study, the median overall survival
(OS) in patients treated with cisplatin/vinorelbine was 52.8 months, which was significantly longer than that of the cisplatin/FU group (25.2 months) (20). However, the pattern of VP neoadjuvant chemotherapy administration varies in hospitals in China, and its optimal dose and intensity in terms of toxicity and efficacy has not yet been determined. VP1 (cisplatin $75 \mathrm{mg} / \mathrm{m}^{2}$ on day 1 ; vinorelbine $25 \mathrm{mg} / \mathrm{m}^{2}$ on days 1 and 8, for two cycles) $(17,18,20)$ and VP2 (cisplatin $25 \mathrm{mg} / \mathrm{m}^{2}$ on days 1 to 4 ; vinorelbine $25 \mathrm{mg} / \mathrm{m}^{2}$ on days 1 and 8, for two cycles) (19) are two commonly used VP regimens for neoadjuvant chemotherapy in ESCC treatment. In order to optimize the dose and intensity of VP neoadjuvant chemotherapy for ESCC, we retrospectively compared the toxicity, postoperative complication rate, and survival among patients with locally advanced ESCC who received either the VP1 or VP2 regimen as neoadjuvant chemotherapy.

We present the following article in accordance with the STROBE reporting checklist (available at http://dx.doi. org/10.21037/atm-21-458).

\section{Methods}

\section{Patients}

Between June 2008 and December 2016, 78 patients with locally advanced esophageal squamous cell cancer who had received VP1 or VP2 for nCRT followed by surgery in Taizhou Hospital of Zhejiang Province were retrospectively reviewed (Table 1).

The inclusion criteria were as follows: (I) patients with histologically confirmed resectable stage IIB or III thoracic ESCC (according to the 6th American Joint Committee on Cancer edition); (II) patients who had not received previous treatment; (III) patients who were expected to have at least 6 months survival; (IV) patients aged between 18 and 70 years; $(\mathrm{V})$ patients with adequate marrow function: white blood cell $\geq 4.0 \times 10^{9} / \mathrm{L}$, neutrophil $\geq 1.5 \times 10^{9} / \mathrm{L}$, platelet $\geq 100.0 \times 10^{9} / \mathrm{L}$, and hemoglobin $\geq 90 \mathrm{~g} / \mathrm{L}$; (VI) patients with normal liver and kidney function; (VII) patients with a Karnofsky performance score $\geq 90$; (VIII) complete clinical data were available. The exclusion criteria were as follows: (I) patients who had received prior treatment for primary tumor or nodes; (II) patients with a history of or concomitant hemorrhagic diseases; (III) pregnancy or lactation; (IV) patients with peripheral neuropathy and the Common Terminology Criteria for Adverse Events, CTCAE v3.0 grade $\geq 2$; (V) patients with prior malignancies, except for adequately treated basal or squamous cell skin 
Table 1 Clinical characteristics of patients receiving different VP regimens

\begin{tabular}{|c|c|c|c|}
\hline Characteristic & $\begin{array}{l}\text { VP1 regimen } \\
\quad(n=47)\end{array}$ & $\begin{array}{l}\text { VP2 regimen } \\
\qquad(n=31)\end{array}$ & $P$ value \\
\hline Age, years & $54.02 \pm 7.18$ & $55.94 \pm 6.07$ & 0.968 \\
\hline Gender & & & 0.153 \\
\hline Male & $42(89.4)$ & $24(77.4)$ & \\
\hline Female & 5 (10.6) & 7 (22.6) & \\
\hline $\mathrm{BMI}, \mathrm{kg} / \mathrm{m}^{2}$ & $22.34 \pm 3.4$ & $22.69 \pm 3.35$ & 0.193 \\
\hline KPS & & & 1.000 \\
\hline 90 & 47 (100.0) & 31 (100.0) & \\
\hline 100 & $0(0)$ & $0(0)$ & \\
\hline Tumor location & & & 0.112 \\
\hline Proximal third & 5 (10.6) & 4 (12.9) & \\
\hline Middle third & $29(61.7)$ & $22(71.0)$ & \\
\hline Distal third & $13(27.3)$ & 5 (16.1) & \\
\hline Clinical T stage & & & 0.757 \\
\hline cT1-T2 & $2(4.3)$ & $5(16.1)$ & \\
\hline сT3 & $22(46.8)$ & $21(67.8)$ & \\
\hline cT4 & 23 (48.9) & 5 (16.1) & \\
\hline Clinical N stage & & & 0.998 \\
\hline NO & $22(46.8)$ & $0(0)$ & \\
\hline $\mathrm{N} 1$ & 25 (53.2) & 31 (100.0) & \\
\hline Clinical stage & & & 0.361 \\
\hline IIB & $2(4.3)$ & $5(16.1)$ & \\
\hline III & 45 (95.7) & 26 (83.9) & \\
\hline CRT cycle & & & 0.057 \\
\hline 1 cycle & 9 (19.1) & $0(0)$ & \\
\hline 2 cycle & 38 (80.2) & $31(100.0)$ & \\
\hline
\end{tabular}

BMI, body mass index; CRT, chemoradiotherapy; KPS, Karnofsky Performance Score; pCR, pathological complete response.

cancer and in situ cervical cancer.

All procedures performed in this study involving human participants were in accordance with the Declaration of Helsinki (as revised in 2013). This study was approved by the medical ethics committee of Taizhou Hospital of Zhejiang Province. All included patients provided signed informed consent.

\section{Neoadjuvant chemotherapy}

Patients received either the VP1 or VP2 chemotherapy regimen every 3 weeks for two cycles. The VP1 regimen was vinorelbine $25 \mathrm{mg} / \mathrm{m}^{2}$, intravenous (IV) bolus, days 1 and 8 and cisplatin $75 \mathrm{mg} / \mathrm{m}^{2}$, IV within 3 hours, day 1 . The VP2 regimen was vinorelbine $25 \mathrm{mg} / \mathrm{m}^{2}$, IV bolus, days 1 and 8 and cisplatin $25 \mathrm{mg} / \mathrm{m}^{2}$, IV within 2 hours on days 1 to 4 .

When the absolute neutrophil count $>1.5 \times 10^{9} / \mathrm{L}$ and the platelet count $\geq 75 \times 10^{9} / \mathrm{L}$, a full-dose of chemotherapy was given. Otherwise, chemotherapy was delayed for up to 2 weeks until the counts recovered. When hematological toxicity persisted for 2 weeks or longer, chemotherapy was discontinued.

\section{Neoadjuvant radiotberapy}

A total dose of 40.0 Gy was administered in 20 fractions of 2.0 Gy, five fractions per week, starting on the first day of the first cycle of chemotherapy. All patients were treated with external beam radiation, using the three-dimensional conformal radiation technique. The gross tumor volume was defined by the primary tumor and any enlarged regional lymph nodes. The clinical target volume provided a proximal and distal margin of $3 \mathrm{~cm}$ and a radial margin of 0.5 to $1.0 \mathrm{~cm}$ radial around the gross tumor volume. The planning target volume provided an 8 - $\mathrm{mm}$ margin of the clinical target volume.

\section{Surgery}

Surgery was performed 4-6 weeks after chemoradiotherapy. Surgery consisted of McKeown or Ivor Lewis esophagectomy, including two-field lymphadenectomy with total mediastinal lymph node dissection. The left and right recurrent laryngeal nerve nodes were mandatorily dissected.

\section{Follow-up}

Post-treatment follow-up was performed once every 3 months within the first year, and every 6 months thereafter until death. The primary endpoint was OS, which was measured from the date of group assignment to the date of death or the last follow-up.

\section{Statistical analysis}

Normally distributed continuous data were expressed as 
Table 2 Overall survival of patients receiving VP1 or VP2 regimen

\begin{tabular}{lccccc}
\hline Regimen & No. (\%) & Overall survival range (months) & Median overall survival $(95 \% \mathrm{Cl})$ & Hazard ratio $(95 \% \mathrm{Cl})$ & $\mathrm{P}$ value \\
\hline VP1 & $47(60.3)$ & $3.63-103.00$ & $-^{\star}$ & 1.000 \\
VP2 & $31(39.7)$ & $4.56-83.90$ & $97.6(85.6-109.7)$ & $1.008(0.999-1.018)$ & 0.509 \\
\hline
\end{tabular}

*, the median overall survival was not reached by the date of data cutoff. $95 \% \mathrm{Cl}, 95 \%$ confidence interval.

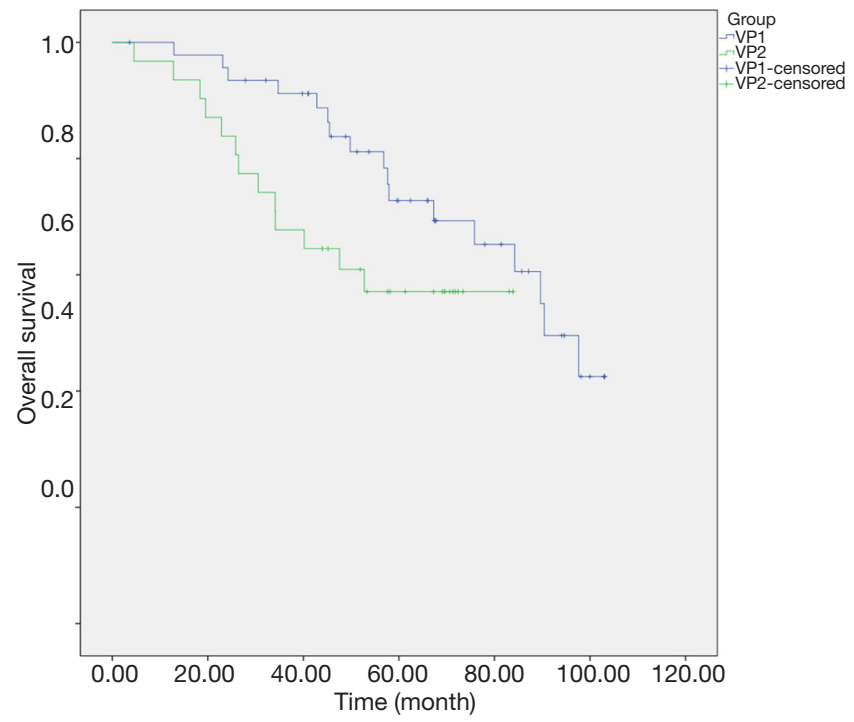

Figure 1 Overall survival curves by group. All values were less than 0.05 .

mean \pm standard deviation $(\mathrm{SD})$. Frequencies were adopted to describe the categorical variables. The demographic and clinical parameters of patients in the VP1 and VP2 groups were compared using the $t$-test for continuous variables and the $\chi^{2}$ test for categorical variables. The Kaplan-Meier method was used to compare OS and disease-free survival (DFS) between the two groups. Statistical analyses were performed using SPSS (IBM SPSS Statistics 19.0, SPSS Inc., Chicago, IL, USA). $\mathrm{P} \leq 0.05$ was considered to be statistically significant.

\section{Results}

\section{Clinical characteristics of the study population}

A total of 78 patients who had received either the VP1 or VP2 regimen chemotherapy plus radiation followed by surgery were included in the present study. There were 47 patients in the VP1 group and 31 patients in the VP2 group (Table 1). The clinical characteristics, including age, gender, tumor location, and clinical stage etc., were not significantly different between the two groups.

\section{Efficacy}

The median OS was 97.6 months (85.6-109.7) in the VP2 group, which was not significantly different with that of the VP1 group [hazard ratio (HR), 1.008 (0.999-1.108); $\mathrm{P}=0.509]$. The median $\mathrm{OS}$ was not reached by the date of data cutoff (Table 2, Figure 1). The OS rate in the VP2 and VP1 groups was $86.4 \%$ and $94.7 \%$ at 1 year, $71.6 \%$ and $79.8 \%$ at 2 years, and $65.4 \%$ and $73.9 \%$ at 3 years, respectively (Table 3).

\section{Safety profile}

The hematologic and non-hematologic toxicity observed during nCRT are listed in Table 4. Hematologic toxicity was common, especially leukopenia and neutropenia. Digestive tract adverse reactions mainly included anorexia, vomiting, and radiation esophagitis, but were mostly grade 1 or 2 .

The rate of all grades of hematologic adverse events, including anemia, leukopenia, neutropenia, and thrombocytopenia, were significantly higher in the VP2 group compared to the VP1 group $(\mathrm{P}<0.05)$. Grade 3 or 4 leukopenia occurred in $27.6 \%$ patients in the VP1 group, which was significantly lower than that of the VP2 group (54.8\%) $(\mathrm{P}=0.047)$. Also, grade 3 or 4 neutropenia was observed in $10.5 \%$ patients in the VP1 group, which was significantly lower than that of the VP2 group (35.5\%) $(\mathrm{P}=0.029)$.

As for non-hematologic toxicity, the rate of all grades of anorexia, vomiting, and fatigue were significantly higher in the VP1 group than those of the VP2 group (all $\mathrm{P}<0.05$ ). However, there was no difference in the grade 3 or 4 nonhematologic adverse events between the two groups (all $\mathrm{P}>0.05)$. No grade 3 or 4 hepatic dysfunction, diarrhea, constipation, or alopecia occurred in either group.

The postoperative complications that occurred in patients of both groups are listed in Table 5. Pulmonary infection, arrhythmia, and anastomotic leakage were 
common in both groups. Pulmonary infection occurred in four patients $(8.5 \%)$ in the VP1 group, and eight patients $(25.8 \%)$ in the VP2 group $(\mathrm{P}=0.038)$. However, other postoperative complications, including hemorrhage, pneumothorax, atelectasis etc., were similar between the two groups (all $\mathrm{P}>0.05$ ).

\section{Discussion}

It is important to determine the optimized dose and intensity of neoadjuvant chemotherapy for EC, especially in terms of toxicity. The VP regimen is an effective and promising treatment combination for ESCC, and is worthy of further exploration (17-20). However, its administration pattern varies in different hospitals of China due to the lack

Table 3 Overall survival rate of patients receiving VP1 or VP2 regimen

\begin{tabular}{llll}
\hline \multirow{2}{*}{ Regimen } & $\begin{array}{l}\text { 1-year OS } \\
(95 \% \mathrm{Cl})(\%)\end{array}$ & $\begin{array}{l}\text { 2-year OS } \\
(95 \% \mathrm{Cl})(\%)\end{array}$ & $\begin{array}{l}\text { 3-year OS } \\
(95 \% \mathrm{Cl})(\%)\end{array}$ \\
\hline VP1 & $94.7(87.8-97.8)$ & $79.8(70.1-86.6)$ & $73.9(63.5-81.7)$ \\
VP2 & $86.4(79.0-91.3)$ & $71.6(62.7-78.7)$ & $65.4(56.2-73.2)$ \\
\hline
\end{tabular}

$95 \% \mathrm{Cl}, 95 \%$ confidence interval. of a standard. Therefore, in this study, we compared the safety and efficacy of two commonly applied dose-intensity VP regimens in patients with locally advanced ESCC.

According to the survival analysis of the present study, the median OS was 97.6 months in patients receiving VP2, with a 3 -year survival rate of $65.4 \%$; the median OS in VP1 group was not obtained, and the 3 -year survival rate was $73.9 \%$. This median OS and 3-year survival rate in the VP2 group were superior to than those reported by Liu et al. in 2015, who noted a median OS of 52.8 months and a 3-year OS rate of $64.3 \%$ among 57 patients with stage $\mathrm{IIb} / \mathrm{III}$ ESCC who also received the VP1 regimen (cisplatin $75 \mathrm{mg} / \mathrm{m}^{2}$ on day 1 ; vinorelbine $25 \mathrm{mg} / \mathrm{m}^{2}$ on days 1 and 8 , for two cycles) (20). The VP1 regimen was also reported as neoadjuvant chemotherapy for locally advanced ESCC patients by Fu et al. (17) and Yang et al. (18), but the survival outcomes for patients receiving the VP1 regimen were not reported. Similarly, the VP2 regimen neoadjuvant chemotherapy had been applied for locally ESCC by Zhu et al., however its benefit on survival was not clear (19). This is the first time that the survival outcomes of patients with locally advanced ESCC who received VP2 nCRT followed by surgery have been reported.

In the present study, the median OS was not significantly

Table 4 Adverse events of patients receiving VP1 or VP2 regimen

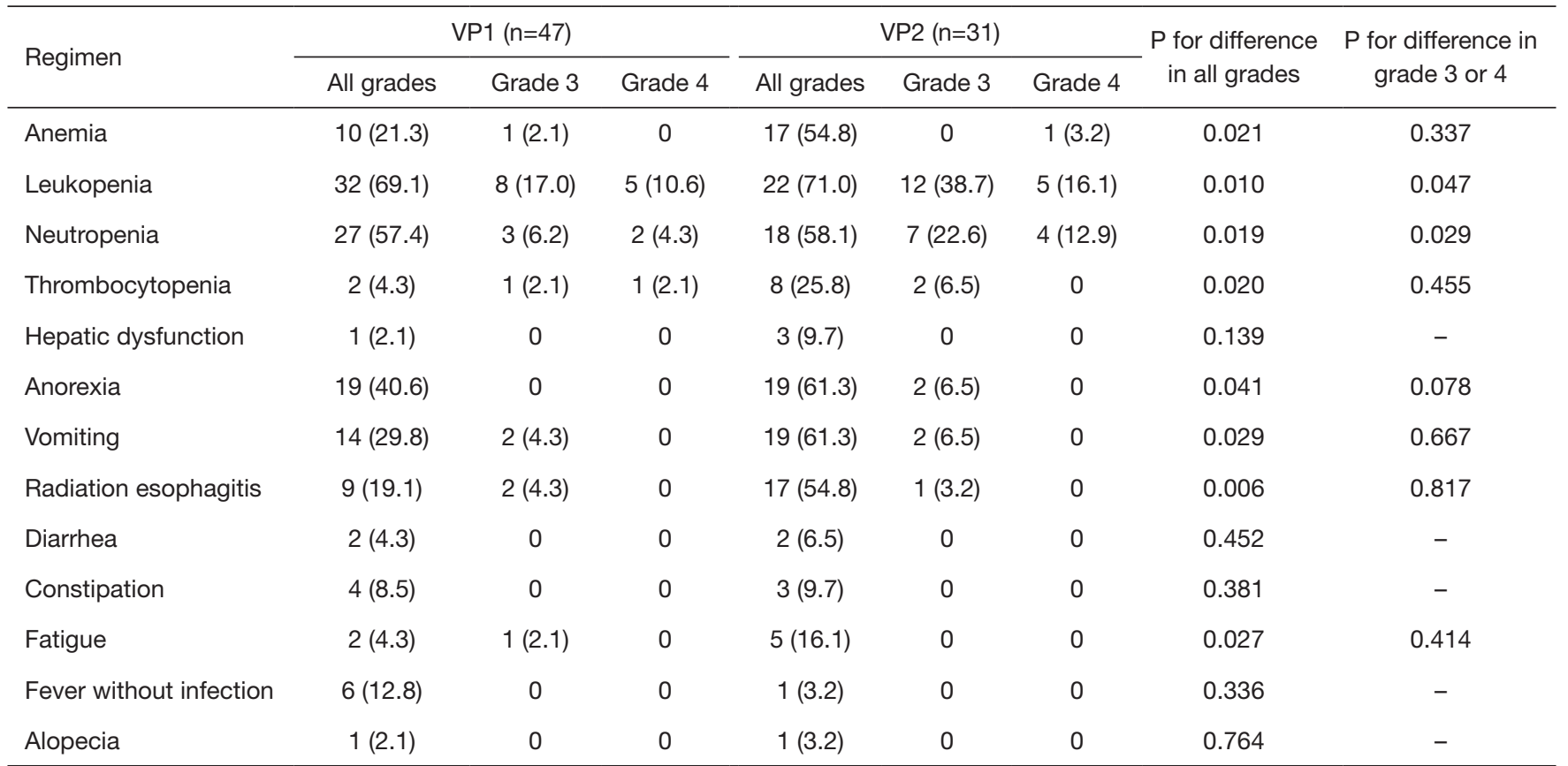

Data presented as No. (\%). Adverse events were graded according to the National Cancer Institute's Common Terminology Criteria for Adverse Events, version 3.0. 
Table 5 Postoperative complications of patients receiving VP1 or VP2 regimen

\begin{tabular}{|c|c|c|c|}
\hline Postoperative complications & $\begin{array}{l}\text { VP1 group } \\
\quad(n=47)\end{array}$ & $\begin{array}{l}\text { VP2 group } \\
\quad(n=31)\end{array}$ & $P$ value \\
\hline Hemorrhage & 0 & 0 & - \\
\hline Pulmonary infection & $4(8.5)$ & $8(25.8)$ & 0.038 \\
\hline Pneumothorax & $2(4.5)$ & $2(6.5)$ & 0.667 \\
\hline Atelectasis & 0 & 0 & - \\
\hline Respiratory failure & 0 & 0 & - \\
\hline Empyema & 0 & 0 & - \\
\hline Arrhythmia & $5(10.6)$ & $4(12.9)$ & 0.759 \\
\hline Heart failure & $1(2.1)$ & $1(3.2)$ & 0.764 \\
\hline Anastomotic leakage & $2(4.3)$ & $3(9.7)$ & 0.339 \\
\hline Gastric fistula & 0 & 0 & - \\
\hline Chylothorax & $1(2.1)$ & 0 & 0.603 \\
\hline Pyloric obstruction & 0 & $1(1.4)$ & 0.414 \\
\hline Intestinal obstruction & $1(2.1)$ & 0 & 0.414 \\
\hline Injury of recurrent nerve & $3(6.4)$ & 0 & 0.151 \\
\hline ARDS & 0 & 0 & - \\
\hline Incision infection & $2(4.3)$ & $1(3.2)$ & 0.817 \\
\hline Fat necrosis of incision & 0 & $1(3.2)$ & 0.215 \\
\hline ACS & 1 & 0 & 0.414 \\
\hline Pleural effusion & 0 & 0 & - \\
\hline Anastomotic stenosis & $3(6.4)$ & 0 & 0.151 \\
\hline
\end{tabular}

Data presented as No. (\%). ARDS, acute respiratory distress syndrome; ACS, acute coronary syndrome.

different between the VP1 and VP2 groups $(\mathrm{P}=0.075)$. The 1-, 2-, and 3-year OS rates were higher in the VP1 group, although the difference was not statistically significant. This result demonstrated that the VP1 and VP2 regimens were comparable in terms of OS.

Previous studies have demonstrated that the VP1 and VP2 regimens were generally well-tolerated, but may lead to myelosuppression, and were thus associated with increased hematologic toxicity $(6,17-20)$. As reported by Liu et al. (20), the incidence of grade 3 or 4 leukopenia and neutropenia related to VP1 were $33.3 \%$ and $31.6 \%$, respectively. Conroy et al. reported that the rate of grade 3 or 4 neutropenia among advanced ESCC patients was $41 \%$ (cisplatin $80 \mathrm{mg} / \mathrm{m}^{2}$ on day 1 ; vinorelbine $25 \mathrm{mg} / \mathrm{m}^{2}$ on days 1 and 8 , for two cycles) (6). These results were similar to our findings for the VP1 regimen. Furthermore, compared with VP1, the VP2 group showed significantly higher rates of all grades of anemia, leukopenia, neutropenia, and thrombocytopenia (all $\mathrm{P}<0.05$ ). Therefore, VP2 exhibited more hematologic toxicity than VP1. Digestive tract adverse events such as anorexia, vomiting, radiation esophagitis etc. were also common, but were mostly grade 1 or 2 . As for the frequency of non-hematologic adverse events of grade 3 or 4, the two groups showed no significant differences (all $\mathrm{P}>0.05$ ). On the whole, VP1 had a better safety profile than VP2, mainly reflected in hematologic toxicity. This result corresponds to the higher compliance rate in the VP1 group, which demonstrated that VP1 confers better tolerance.

Pulmonary infection, arrhythmia, and anastomotic leakage were the most common postoperative complications in our study. This is consistent with the study performed by Liu et al. (20) in which pulmonary complications, cardiac complications, and anastomotic leakage were the top three most common surgical complications. Of all the surgical complications, the frequency of pulmonary infection was significantly higher in the VP2 group compared to the VP1 group $(25.8 \%$ vs. $8.5 \%, \mathrm{P}=0.038)$, while the frequencies of other complication were similar between the two groups (all $\mathrm{P}>0.05$ ). This result may be associated with the more severe myelosuppression in the VP2 group, as indicated by higher rates of leukopenia and neutropenia.

In summary, VP1 nCRT is comparable with VP2 in terms of survival among locally advanced ESCC patients. However, it has better compliance, tolerance, and safety profile. Therefore, we recommend the use of VP1 over VP2 for neoadjuvant chemotherapy in ESCC.

There are several limitations in our study that should be noted. Firstly, this is a retrospective cohort study, which, in contrast to a prospective randomized control study, exhibits selection bias. However, the clinical characteristics between two groups were not significantly different. Secondly, all of the included patients were diagnosed with an ESCC subtype, and thus the results may not be generalizable to the EC population, including the adenocarcinoma subtype.

\section{Conclusions}

The VP1 regimen showed comparable efficacy to the VP2 regimen in terms of survival, but was associated with better compliance, as well as less hematologic toxicity and postoperative pulmonary infection. Therefore, we recommend VP1 over VP2 to be the optimized VP 
neoadjuvant chemotherapy regimen for locally advanced ESCC.

\section{Acknowledgments}

Funding: None.

\section{Footnote}

Reporting Checklist: The authors have completed the STROBE reporting checklist. Available at http://dx.doi. org/10.21037/atm-21-458

Data Sharing Statement: Available at http://dx.doi. org/10.21037/atm-21-458

Conflicts of Interest: All authors have completed the ICMJE uniform disclosure form (available at http://dx.doi. org/10.21037/atm-21-458). The authors have no conflicts of interest to declare.

Ethical Statement: The authors are accountable for all aspects of the work in ensuring that questions related to the accuracy or integrity of any part of the work are appropriately investigated and resolved. All procedures performed in this study involving human participants were in accordance with the Declaration of Helsinki (as revised in 2013). This study was approved by the ethics committee of Taizhou Hospital of Zhejiang Province Affiliated to Wenzhou Medical University. All included patients provided signed informed consent.

Open Access Statement: This is an Open Access article distributed in accordance with the Creative Commons Attribution-NonCommercial-NoDerivs 4.0 International License (CC BY-NC-ND 4.0), which permits the noncommercial replication and distribution of the article with the strict proviso that no changes or edits are made and the original work is properly cited (including links to both the formal publication through the relevant DOI and the license). See: https://creativecommons.org/licenses/by-nc-nd/4.0/.

\section{References}

1. Pakzad R, Mohammadian-Hafshejani A, Ghoncheh M, et al. The incidence and mortality of lung cancer and their relationship to development in Asia. Transl Lung Cancer Res 2015;4:763-74.
2. Donahue JM, Nichols FC, Li Z, et al. Complete pathologic response after neoadjuvant chemoradiotherapy for esophageal cancer is associated with enhanced survival. Ann Thorac Surg 2009;87:392-8.

3. Sjoquist KM, Burmeister BH, Smithers BM, et al. Survival after neoadjuvant chemotherapy or chemoradiotherapy for resectable oesophageal carcinoma: An updated metaanalysis. Lancet Oncol 2011;12:681-92.

4. Gebski V, Burmeister B, Smithers BM, et al. Survival benefits from neoadjuvant chemoradiotherapy or chemotherapy in oesophageal carcinoma: a meta-analysis. Lancet Oncol 2007;8:226-34.

5. Zhang CD, Zeng YJ, Li HW, et al. Neoadjuvant chemotherapy for nonmetastatic esophago-gastric adenocarcinomas: a systematic review and meta-analysis. Cancer Invest 2013;31:421-31.

6. Conroy T, Etienne PL, Adenis A, et al. Vinorelbine and cisplatin in metastatic squamous cell carcinoma of the oesophagus: response, toxicity, quality of life and survival. Ann Oncol 2002;13:721-9.

7. Bleiberg H, Conroy T, Paillot B, et al. Randomised phase II study of cisplatin and 5-fluorouracil (5-FU) versus cisplatin alone in advanced squamous cell oesophageal cancer. Eur J Cancer 1997;33:1216-20.

8. Ilson DH, Forastiere A, Arquette M, et al. A phase II trial of paclitaxel and cisplatin in patients with advanced carcinoma of the esophagus. Cancer J 2000;6:316-23.

9. Fiorica F, Di Bona D, Schepis F, et al. Preoperative chemoradiotherapy for oesophageal cancer: a systematic review and meta-analysis. Gut 2004;53:925-30.

10. Levard H, Pouliquen X, Hay JM, et al. 5-Fluorouracil and cisplatin as palliative treatment of advanced oesophageal squamous cell carcinoma. A multicentre randomised controlled trial. Eur J Surg 1998;164:849-57.

11. Matsumoto A, Nishikawa K, Yuda M, et al. Early response of esophageal cancer to neoadjuvant chemotherapy with docetaxel-cisplatin-5-fluorouracil represents sensitivity: a phase II study. Anticancer Res 2016;36:1937-42.

12. Ku GY. Systemic therapy for esophageal cancer: chemotherapy. Chin Clin Oncol 2017;6:49.

13. Enzinger PC, Ilson DH, Kelsen DP. Chemotherapy in esophageal cancer. Semin Oncol 1999;26:12-20.

14. Conroy T, Etienne PL, Adenis A, et al. Phase II trial of vinorelbine in metastatic squamous cell esophageal carcinoma. J Clin Oncol 1996;14:164-70.

15. Bidoli P, Stani SC, De Candis D, et al. Single-agent chemotherapy with vinorelbine for pretreated or metastatic 
squamous cell carcinoma of the esophagus. Tumori 2001;87:299-302.

16. Ilson DH, Kelsen DP. Management of esophageal cancer. Oncology 1996;10:1385-96.

17. Fu JH, Rong TH, Li XD, et al. Chemoradiotherapy followed by surgery in treatment of locally advanced esophageal carcinoma: a phase II trial. Ai Zheng 2004;23:1473-6.

18. Yang H, Fu JH, Hu Y, et al. Neo-adjuvant chemoradiotherapy followed by surgery in treatment of advanced esophageal carcinoma. Zhonghua Yi Xue Za Zhi 2008;88:3182-5

Cite this article as: Jin K, Chen B, Wang C, Zhang B, Zhang J, Kong M, Wang L, Zhu C, Shen J. Efficacy and safety of vinorelbine and cisplatin regimen of different doses and intensities for neoadjuvant chemotherapy in patients with locally advanced esophageal carcinoma. Ann Transl Med 2021;9(8):660. doi: 10.21037/atm-21-458
19. Zhu CC, Chen BF, Kong M, et al. Neoadjuvant chemoradiotherapy followed by combined thoracoscopic and laparoscopic esophagectomy in the treatment of locally advanced esophageal carcinoma. Zhonghua Wei Chang Wai Ke Za Zhi 2012;15:943-6.

20. Liu SL, Yang H, Zhang P, et al. Neoadjuvant chemoradiotherapy with cisplatin plus vinorelbine versus cisplatin plus fluorouracil for esophageal squamous cell carcinoma: a matched case-control study. Radiother Oncol 2015;116:262-8.

(English Language Editor: A. Kassem) 